\title{
铜(I)催化偕二取代芳香烯烃氧化成酮的反应研究
}

\author{
徐星关耀辉徐东成李新生*
}

(浙江师范大学化学与生命科学学院 金华 321004)

摘要 以吡啶为溶剂, 溴化亚铜作催化剂, 催化偕二取代烯烃氧化成酮, 并获得良好的产率( $31 \%$ \% $95 \%)$. 该反应具有 操作简单、催化剂量少、选择性及产率高等优点, 为芳香端烯的断键氧化提供了好的选择.

关键词 溴化亚铜; 偕二取代烯烃; 氧化成酮

\section{Copper(I)-Catalyzed Oxidation of Disubstituted Aromatic Alkenes to Ketones}

\author{
Xu, Xing Guan, Yaohui $\quad \mathrm{Xu}$, Dongcheng Li, Xinsheng* \\ (College of Chemistry and Life Science, Zhejiang Normal University, Jinhua 321004)
}

\begin{abstract}
Oxidation of 1,1-disubstituted alkenes to ketones in pyridine with $\mathrm{CuBr}$ as catalyst in high yields (31\% 95\%) was discovered. The reaction, simple operation, low catalyst loading, excellent selectivity and yields provided a good choice for the oxidative cleavage of aromatic terminal olefins to ketones.
\end{abstract}

Keywords $\mathrm{CuBr}$; disubstituted alkene; oxidation; ketone; catalyst

烯烃的氧化在有机合成中具有重要的作用, 对合成 药物中间体来说也是关键的一步. 烯烃除了可以氧化成 邻二醇 ${ }^{[1]}$ 和环氧类 ${ }^{[2]}$ 化合物外, 将其断键氧化成醛酮类 化合物也是其重要的一类反应. 目前已经有很多方法能 完成这个反应, 其中, 臭氧氧化就是经典的一种 ${ }^{[3]}$, 然 而, 出于安全性的考虑, 这种方法的运用和发展都受到 了限制 ${ }^{[4]}$. 很多过渡金属, 如钓 ${ }^{[5]}$ 、锇 ${ }^{[6]}$ 、锰 ${ }^{[7]}$ 、铇 ${ }^{[8]}$ 、 铼 ${ }^{[9]} 、 \mathrm{c}^{[10]}$ 、铁 ${ }^{[11]} 、$ 金 $^{[12]}$ 和铜 $^{[13]}$ 也被应用于这类反应, 还 有无金属催化的方法也已有报道 ${ }^{[14]}$. 尽管目前的烯烃 断键氧化已经取得了很大的成就, 但是简便操作, 经济 高效的氧化方法仍需进行探索.

\section{1 结果与讨论}

\section{1 反应条件的优化}

以 1,1 -二苯乙烯为原料, 溴化亚铜为催化剂进行反 应条件的优化. 实验探索了各类含氮配体及其用量(图 1)、温度、溶剂、氧化剂等因素对反应的影响.<smiles>c1ccncc1</smiles>

$\mathrm{L}_{1}$

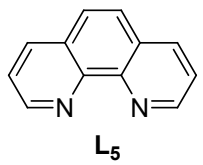<smiles>c1ccc(-c2ccccn2)nc1</smiles>

$\mathrm{L}_{2}$<smiles>Oc1cccc2cccnc12</smiles>

$\mathrm{L}_{3}$<smiles>CN(C)CC(=O)O</smiles>

$\mathrm{L}_{4}$<smiles>COc1ccnc2c1ccc1c(OC)ccnc12</smiles>

图 1 配体 $\mathbf{L}_{1} \sim \mathbf{L}_{7}$

Figure 1 Ligands $\mathbf{L}_{1} \sim \mathbf{L}_{7}$

反应结果见表 1 . 以甲苯为溶剂, 我们对不同的含 氮配体进行一系列的笁选(表 1, Entries 1 7), 发现吡啶 和联吡啶 ${ }^{[15]}$ 在作配体时都具有良好的产率，考虑到成 本因素, 我们选择吡啶作配体, 且当其用量为 0.1 equiv. 时, 反应已达到好的效果(表 1, Entries 1，8，9). 在反应 过程中, 我们发现少量水对反应影响较大(表 1, Entry 10), 当向体系中加入 $0.05 \mathrm{~mL}$ 水时, 氧化产率明显提高. 除甲苯外, 我们也尝试了其他溶剂, 包括吡啶、乙醇、

\footnotetext{
*E-mail: ssky33@zjnu.cn

Received September 26, 2015; revised December 11, 2015; published online December 15, 2015.

Project supported by the Master Innovation Training Project of Zhejiang Province.

浙江省化学重中之重研究生创新训练资助项目.
} 
甲醇、四氢呋喃等(表 1, Entries 14 21), 其中, 吡啶作 溶剂时, 氧化产率高达 93\%. 对催化剂而言, 不同的铜 (I)盐对反应的催化效果也不同(表 1, Entries 14, 22 23), 其中, 溴化亚铜的催化效果最好. 氧化剂对反应影响很 大(表 1, Entries $11 \sim 14,19,24 \sim 25)$, 过氧化叔丁醇 (TBHP)比二叔丁基过氧化物(DTBP)在反应中表现出更 好的氧化能力, 且当其用量为 3.0 equiv. 时, 氧化效果最 佳. 温度对反应的影响较为显著(表 1, Entries 14, 26 27), 温度越高, 反应越快, 产率也越高.

\section{2 底物拓展}

为了探索该反应的适用范围, 在上述最佳反应条件 下，我们选择偕二取代的烯烃进行研究.

实验结果表明(表 2), 苯环上取代基的电子效应对 该反应影响不大，吸电子基可以提高反应速率(表 2 ,
Entries 1 12), 对位取代比间位取代反应活性更高，产 率更高(表 2, Entries 1, 2, 5, 6, 9, 10). 当把其中一个芳基 换成甲基，反应能够顺利进行，但反应速率变慢，产率 降低(表 2, Entries 13～18). 当把 1,1-二取代换成 1,2-二 取代或单取代烯烃时，反应不能进行(表 2, Entries 19, 20). 烷基取代的烯烃不发生该反应(表 2, Entry 21). 底 物的空间位阻对该反应影响较大(表 2, Entries 3, 22 23), 空间位阻增大，使该反应产率降低.

\section{2 结论}

以铜(I)催化的具有特殊结构的偕二取代端烯氧化 成酮，该反应具有较高的选择性和良好的反应产率，高 效、简便地获得相对应的酮类化合物, 避免了昂贵配体 的使用，是一种不错的氧化方法.

表 1 不同条件对反应的影响 ${ }^{a}$

Table1 The effect of different reaction parameters

\begin{tabular}{|c|c|c|c|c|c|c|c|}
\hline Entry & Solvent & Temp $/{ }^{\circ} \mathrm{C}$ & Cat (equiv) & L (equiv) & Water/mL & Oxidant (eauiv) & Yield ${ }^{b} / 0$ \\
\hline 1 & & & & & & & \\
\hline & 10iuente & 00 & CUDI (0.0J) & $\mathrm{L}_{1}(0.1)$ & - & 1ВНР (2.0) & 58 \\
\hline 2 & Toluene & 80 & $\mathrm{CuBr}(0.05)$ & $\mathbf{L}_{2}(0.1)$ & - & TBHP (2.0) & 59 \\
\hline 3 & Toluene & 80 & $\mathrm{CuBr}(0.05)$ & $\mathbf{L}_{3}(0.1)$ & - & TBHP (2.0) & 54 \\
\hline 4 & Toluene & 80 & $\mathrm{CuBr}(0.05)$ & $\mathbf{L}_{4}(0.1)$ & - & TBHP (2.0) & 35 \\
\hline 5 & Toluene & 80 & $\mathrm{CuBr}(0.05)$ & $\mathbf{L}_{5}(0.1)$ & - & TBHP (2.0) & 26 \\
\hline 6 & Toluene & 80 & $\mathrm{CuBr}(0.05)$ & $\mathbf{L}_{6}(0.1)$ & - & TBHP (2.0) & 47 \\
\hline 7 & Toluene & 80 & $\mathrm{CuBr}(0.05)$ & $\mathbf{L}_{7}(0.1)$ & - & TBHP (2.0) & 57 \\
\hline 8 & Toluene & 80 & $\mathrm{CuBr}(0.05)$ & $\mathbf{L}_{1}(0.05)$ & - & TBHP (2.0) & 38 \\
\hline 9 & Toluene & 80 & $\mathrm{CuBr}(0.05)$ & $\mathbf{L}_{1}(0.15)$ & - & TBHP (2.0) & 59 \\
\hline 10 & Toluene & 80 & $\mathrm{CuBr}(0.05)$ & $\mathbf{L}_{1}(0.1)$ & 0.05 & TBHP (2.0) & 71 \\
\hline 11 & Toluene & 80 & $\mathrm{CuBr}(0.05)$ & $\mathbf{L}_{1}(0.1)$ & 0.05 & TBHP (1.0) & 49 \\
\hline 12 & Toluene & 80 & $\mathrm{CuBr}(0.05)$ & $\mathbf{L}_{1}(0.1)$ & 0.05 & TBHP (3.0) & 82 \\
\hline 13 & Toluene & 80 & $\mathrm{CuBr}(0.05)$ & $\mathbf{L}_{1}(0.1)$ & 0.05 & TBHP (4.0) & 82 \\
\hline 14 & Pyridine & 80 & $\mathrm{CuBr}(0.05)$ & - & 0.05 & TBHP (3.0) & 93 \\
\hline 15 & $\mathrm{EtOH}$ & 80 & $\mathrm{CuBr}(0.05)$ & $\mathbf{L}_{1}(0.1)$ & 0.05 & TBHP (3.0) & 42 \\
\hline 16 & $\mathrm{CH}_{3} \mathrm{OH}$ & 80 & $\mathrm{CuBr}(0.05)$ & $\mathbf{L}_{1}(0.1)$ & 0.05 & TBHP (3.0) & 63 \\
\hline 17 & $\mathrm{C}\left(\mathrm{CH}_{3}\right)_{3} \mathrm{OH}$ & 80 & $\mathrm{CuBr}(0.05)$ & $\mathbf{L}_{1}(0.1)$ & 0.05 & TBHP (3.0) & 78 \\
\hline 18 & THF & 80 & $\mathrm{CuBr}(0.05)$ & $\mathbf{L}_{1}(0.1)$ & 0.05 & TBHP (3.0) & 37 \\
\hline 19 & $30 \% \mathrm{H}_{2} \mathrm{O}_{2}$ & 80 & $\mathrm{CuBr}(0.05)$ & $\mathbf{L}_{1}(0.1)$ & - & - & 36 \\
\hline 20 & $\mathrm{H}_{2} \mathrm{O}$ & 80 & $\mathrm{CuBr}(0.05)$ & $\mathbf{L}_{1}(0.1)$ & - & TBHP (3.0) & 39 \\
\hline 21 & - & 80 & $\mathrm{CuBr}(0.05)$ & $\mathbf{L}_{1}(0.1)$ & 0.05 & TBHP (3.0) & 47 \\
\hline 22 & Pyridine & 80 & $\mathrm{CuCl}(0.05)$ & - & 0.05 & TBHP (3.0) & 79 \\
\hline 23 & Pyridine & 80 & $\mathrm{CuI}(0.05)$ & - & 0.05 & TBHP (3.0) & 89 \\
\hline 24 & Pyridine & 80 & $\mathrm{CuBr}(0.05)$ & - & 0.05 & DTBP (3.0) & 67 \\
\hline 25 & Pyridine & 80 & $\mathrm{CuBr}(0.05)$ & - & 0.05 & - & - \\
\hline 26 & Pyridine & 30 & $\mathrm{CuBr}(0.05)$ & - & 0.05 & ТВHР (3.0) & 78 \\
\hline 27 & Pyridine & 55 & $\mathrm{CuBr}(0.05)$ & - & 0.05 & TBHP (3.0) & 82 \\
\hline 28 & Pyridine & 80 & $\mathrm{CuBr}(0.02)$ & - & 0.05 & TBHP (3.0) & 68 \\
\hline 29 & Pyridine & 80 & $\mathrm{CuBr}(0.08)$ & 一 & 0.05 & TBHP (3.0) & 93 \\
\hline
\end{tabular}

的应条件: 烯烃 $(0.5 \mathrm{mmol})$, 催化剂 $(0.025 \mathrm{mmol})$, 反应温度 $80{ }^{\circ} \mathrm{C}$, 反应时间 $12 \mathrm{~h}$, 溶剂 $1.5 \mathrm{~mL} ;{ }^{b}$ 分离产率. 
表 2 不同底物的氧化反应考察 ${ }^{a}$

Table 2 Oxidation of different substrates

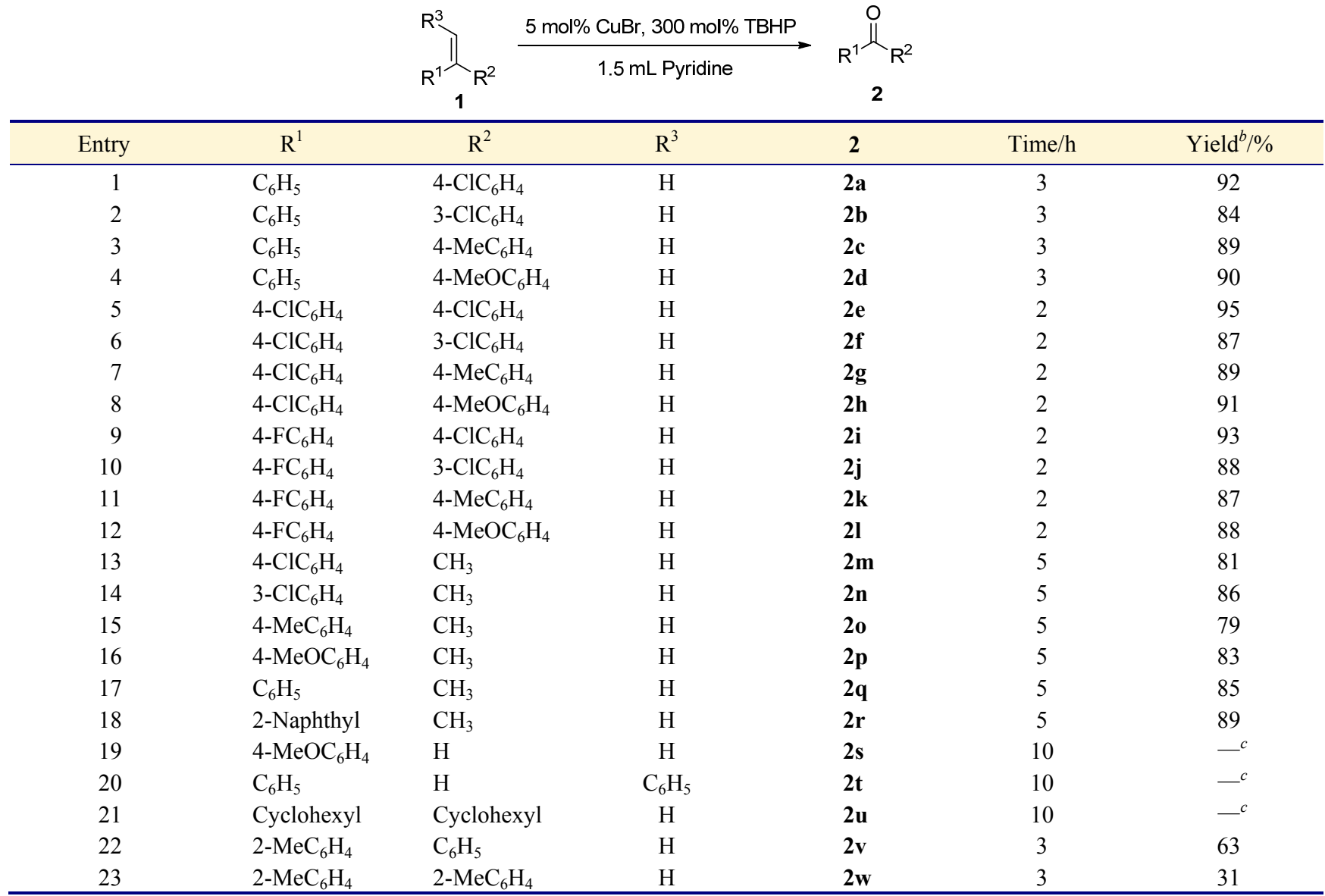

辰应条件: 烯烃 $(0.5 \mathrm{mmol}), \mathrm{CuBr}(0.025 \mathrm{mmol}), \mathrm{TBHP}\left(0.3 \mathrm{~mL}, 5 \mathrm{~mol} \cdot \mathrm{L}^{-1}\right.$ in decane $)$, 反应温度 $80{ }^{\circ} \mathrm{C}$, 吡啶 $(1.5 \mathrm{~mL})$, 水 $(0.05 \mathrm{~mL}) ;^{b}$ 分离产率; ${ }^{c}$ 加热摚拌, 无反应发生

\section{3 实验部分}

\section{1 仪器与试剂}

Bruker600M 核磁共振仪(以 $\mathrm{TMS}$ 为内标, $\mathrm{CDCl}_{3}$ 为 溶剂); 数字显示微熔点测定仪; 试剂均为市售分析纯 或化学纯.

\section{2 实验方法}

在反应试管中加入芳香烯烃 $1(0.5 \mathrm{mmol})$ 、催化剂 $\mathrm{CuBr}(0.025 \mathrm{mmol}) 、 \mathrm{TBHP}\left(0.3 \mathrm{~mL}, 5 \mathrm{~mol} \cdot \mathrm{L}^{-1}\right.$ in decane 、水 $(0.05 \mathrm{~mL})$ 和吡啶 $(1.5 \mathrm{~mL})$, 放置 $80{ }^{\circ} \mathrm{C}$ 的油浴锅 中进行反应, TLC 跟踪反应进程, 当原料反应完全后, 停止反应, 转移至 $150 \mathrm{~mL}$ 分液漏斗中, 二氯甲烷萃取 (20 $\mathrm{mL} \times 3)$, 合并有机相, 旋干, 硅胶柱层析分离纯化, 即可得到产物 2 .

4-氯二苯甲酮 $(\mathbf{2 a})^{[16]}$ : 白色固体, 产率 $92 \%$. m.p. $77 \sim 78{ }^{\circ} \mathrm{C} ;{ }^{1} \mathrm{H}$ NMR $\left(600 \mathrm{MHz}, \mathrm{CDCl}_{3}\right) \delta: 7.77 \sim 7.72$ $(\mathrm{m}, 4 \mathrm{H}), 7.640 \sim 7.57(\mathrm{~m}, 1 \mathrm{H}), 7.49 \sim 7.44(\mathrm{~m}, 4 \mathrm{H}) ;{ }^{13} \mathrm{C}$ NMR $\left(150 \mathrm{MHz}, \mathrm{CDCl}_{3}\right) \delta: 195.5,138.9,137.3,135.9$, 132.7, 131.5, 129.9, 128.7, 128.4.
3-氯二苯甲酮 $(\mathbf{2 b})^{[16]}$ : 白色固体, 产率 $84 \%$. m.p. $83 \sim 84{ }^{\circ} \mathrm{C} ;{ }^{1} \mathrm{H}$ NMR $\left(600 \mathrm{MHz}, \mathrm{CDCl}_{3}\right) \delta: 7.80 \sim 7.77$ $(\mathrm{m}, 3 \mathrm{H}), 7.68 \sim 7.65(\mathrm{~m}, 1 \mathrm{H}), 7.63 \sim 7.59(\mathrm{~m}, 1 \mathrm{H}), 7.58 \sim$ $7.54(\mathrm{~m}, 1 \mathrm{H}), 7.50(\mathrm{t}, J=7.8 \mathrm{~Hz}, 2 \mathrm{H}), 7.42(\mathrm{t}, J=7.8 \mathrm{~Hz}$, $1 \mathrm{H}) ;{ }^{13} \mathrm{C}$ NMR $\left(150 \mathrm{MHz}, \mathrm{CDCl}_{3}\right) \delta: 195.3,139.3,136.9$, 134.6, 132.9, 132.4, 130.0, 129.9, 129.7, 128.5, 128.1.

4-甲基二苯甲酮(2c) ${ }^{[17]}$ : 白色固体, 产率 $89 \%$. m.p. $59 \sim 60{ }^{\circ} \mathrm{C} ;{ }^{1} \mathrm{H}$ NMR (600 MHz, $\left.\mathrm{CDCl}_{3}\right) \delta: 7.77$ (d, $J=7.2$ $\mathrm{Hz}, 2 \mathrm{H}), 7.71$ (d, $J=8.1 \mathrm{~Hz}, 2 \mathrm{H}), 7.55$ (t, $J=7.4 \mathrm{~Hz}, 1 \mathrm{H})$, 7.45 (t, $J=7.7 \mathrm{~Hz}, 2 \mathrm{H}), 7.26$ (d, $J=8.2 \mathrm{~Hz}, 2 \mathrm{H}), 2.42$ (s, $3 \mathrm{H}) ;{ }^{13} \mathrm{C}$ NMR $\left(150 \mathrm{MHz}, \mathrm{CDCl}_{3}\right) \delta: 196.5,143.3,137.9$, $134.9,132.2,130.3,129.9,129.0,128.3,21.7$.

4-甲氧基二苯甲酮 $(2 d)^{[16]}$ : 淡黄色固体，产率 $90 \%$. m.p. $61 \sim 62{ }^{\circ} \mathrm{C} ;{ }^{1} \mathrm{H}$ NMR $\left(600 \mathrm{MHz}, \mathrm{CDCl}_{3}\right) \delta$ : $7.82(\mathrm{~d}$, $J=8.8 \mathrm{~Hz}, 2 \mathrm{H}), 7.74(\mathrm{~d}, J=7.0 \mathrm{~Hz}, 2 \mathrm{H}), 7.56 \sim 7.52(\mathrm{~m}$, $1 \mathrm{H}), 7.45$ (t, $J=7.7 \mathrm{~Hz}, 2 \mathrm{H}), 6.95$ (d, $J=8.9 \mathrm{~Hz}, 2 \mathrm{H}), 3.85$ $(\mathrm{s}, 3 \mathrm{H}) ;{ }^{13} \mathrm{C} \mathrm{NMR}\left(150 \mathrm{MHz}, \mathrm{CDCl}_{3}\right) \delta: 195.5,163.3$, 138.3, 132.6, 131.9, 130.1, 129.7, 128.2, 113.6, 55.5.

4,4'-二氯二苯甲酮 $(2 \mathrm{e})^{[17]}$ : 白色固体，产率 $95 \%$. 
m.p. $147 \sim 148{ }^{\circ} \mathrm{C} ;{ }^{1} \mathrm{HNMR}\left(600 \mathrm{MHz}, \mathrm{CDCl}_{3}\right) \delta: 7.63(\mathrm{~d}$, $J=8.5 \mathrm{~Hz}, 4 \mathrm{H}), 7.37(\mathrm{~d}, J=8.5 \mathrm{~Hz}, 4 \mathrm{H}) ;{ }^{13} \mathrm{C}$ NMR $(150$ $\mathrm{MHz}, \mathrm{CDCl}_{3}$ ) $\delta: 194.2,139.2,135.5,131.3,128.8$.

$3,4^{\prime}$-二氯二苯甲酮 $(2 \mathrm{f})^{[16]}$ : 淡黄色固体, 产率 $87 \%$. m.p. $112 \sim 113^{\circ} \mathrm{C} ;{ }^{1} \mathrm{H}$ NMR $\left(600 \mathrm{MHz}, \mathrm{CDCl}_{3}\right) \delta: 7.74(\mathrm{~d}$, $J=8.6 \mathrm{~Hz}, 3 \mathrm{H}), 7.64 \sim 7.62(\mathrm{~m}, 1 \mathrm{H}), 7.58 \sim 7.56(\mathrm{~m}, 1 \mathrm{H})$, $7.47(\mathrm{~d}, J=8.6 \mathrm{~Hz}, 2 \mathrm{H}), 7.43(\mathrm{t}, J=7.8 \mathrm{~Hz}, 1 \mathrm{H}) ;{ }^{13} \mathrm{C} \mathrm{NMR}$ $\left(150 \mathrm{MHz}, \mathrm{CDCl}_{3}\right) \delta: 194.0,139.4,138.9,135.2,134.7$, 132.6, 131.4, 129.8, 129.8, 128.9, 128.0.

4-氯-4'-甲基二苯甲酮 $(2 \mathrm{~g})^{[17]}$ : 白色固体, 产率 $89 \%$. m.p. $128 \sim 129{ }^{\circ} \mathrm{C} ;{ }^{1} \mathrm{H}$ NMR $\left(600 \mathrm{MHz}, \mathrm{CDCl}_{3}\right) \delta: 7.72$ (d, $J=8.5 \mathrm{~Hz}, 2 \mathrm{H}), 7.68$ (d, $J=8.1 \mathrm{~Hz}, 2 \mathrm{H}), 7.43$ (d, $J=$ $8.4 \mathrm{~Hz}, 2 \mathrm{H}), 7.27$ (d, $J=8.0 \mathrm{~Hz}, 2 \mathrm{H}), 2.43(\mathrm{~s}, 3 \mathrm{H}) ;{ }^{13} \mathrm{C}$ NMR $\left(150 \mathrm{MHz}, \mathrm{CDCl}_{3}\right) \delta: 195.2,143.5,138.6,136.2$, 134.5, 131.4, 130.2, 129.1, 128.6, 21.7.

4-氯-4'-甲氧基二苯甲酮 $(\mathbf{2 h})^{[16]}$ : 白色固体, 产率 91\%. m.p. 124 $125{ }^{\circ} \mathrm{C} ;{ }^{1} \mathrm{H}$ NMR (600 MHz, $\left.\mathrm{CDCl}_{3}\right) \delta$ : $7.79(\mathrm{~d}, J=8.9 \mathrm{~Hz}, 2 \mathrm{H}), 7.70$ (d, $J=8.6 \mathrm{~Hz}, 2 \mathrm{H}), 7.44$ (d, $J=8.6 \mathrm{~Hz}, 2 \mathrm{H}), 6.96$ (d, $J=8.9 \mathrm{~Hz}, 2 \mathrm{H}), 3.88(\mathrm{~s}, 3 \mathrm{H}) ;{ }^{13} \mathrm{C}$ NMR $\left(150 \mathrm{MHz}, \mathrm{CDCl}_{3}\right) \delta: 194.2,163.4,138.3,136.6$, 132.5, 131.2, 129.8, 128.5, 113.7, 55.5.

4-氯-4'-氟二苯甲酮 $(2 \mathbf{i})^{[17]}$ : 白色固体, 产率 $93 \%$. m.p. $114 \sim 115{ }^{\circ} \mathrm{C} ;{ }^{1} \mathrm{H}$ NMR (600 MHz, $\left.\mathrm{CDCl}_{3}\right) \delta: 7.83 \sim$ $7.80(\mathrm{~m}, 2 \mathrm{H}), 7.73 \sim 7.71(\mathrm{~m}, 2 \mathrm{H}), 7.47 \sim 7.45(\mathrm{~m}, 2 \mathrm{H})$, $7.18 \sim 7.15(\mathrm{~m}, 2 \mathrm{H}) ;{ }^{13} \mathrm{C}$ NMR $\left(150 \mathrm{MHz}, \mathrm{CDCl}_{3}\right) \delta$ : $194.0,165.5$ (d, $J=253.2 \mathrm{~Hz}, 1 \mathrm{C}), 134.0,135.8,133.5$, $132.6(\mathrm{~d}, J=9.2 \mathrm{~Hz}, 1 \mathrm{C}), 131.3,128.7,115.6$ (d, $J=21.9$ $\mathrm{Hz}, 1 \mathrm{C})$.

3-氯-4'-氟二苯甲酮 $(\mathbf{2 j})^{[21]}$ : 白色固体, 产率 $88 \%$. m.p. $126 \sim 127{ }^{\circ} \mathrm{C} ;{ }^{1} \mathrm{H}$ NMR $\left(600 \mathrm{MHz}, \mathrm{CDCl}_{3}\right) \delta: 7.85 \sim$ $7.81(\mathrm{~m}, 2 \mathrm{H}), 7.74(\mathrm{t}, J=1.7 \mathrm{~Hz}, 1 \mathrm{H}), 7.63(\mathrm{~d}, J=7.7 \mathrm{~Hz}$, $1 \mathrm{H}), 7.58 \sim 7.54(\mathrm{~m}, 1 \mathrm{H}), 7.43(\mathrm{t}, J=7.8 \mathrm{~Hz}, 1 \mathrm{H}), 7.17(\mathrm{t}$, $J=8.6 \mathrm{~Hz}, 2 \mathrm{H}) ;{ }^{13} \mathrm{C} \mathrm{NMR}\left(150 \mathrm{MHz}, \mathrm{CDCl}_{3}\right) \delta: 193.7$, 165.6 (d, $J=253.5 \mathrm{~Hz}, 1 \mathrm{C}), 139.2,134.7,133.2,132.7$ (d, $J=9.2 \mathrm{~Hz}, 1 \mathrm{C}), 132.4,129.7,127.9,115.7$ (d, $J=21.8 \mathrm{~Hz}$, $1 \mathrm{C})$.

4-氟-4'-甲基二苯甲酮(2k) ${ }^{[17]}$ : 白色固体，产率 $87 \%$. m.p. $98 \sim 99{ }^{\circ} \mathrm{C} ;{ }^{1} \mathrm{H}$ NMR $\left(600 \mathrm{MHz}, \mathrm{CDCl}_{3}\right) \delta: 7.83 \sim$ $7.81(\mathrm{~m}, 2 \mathrm{H}), 7.68(\mathrm{~d}, J=8.1 \mathrm{~Hz}, 2 \mathrm{H}), 7.28(\mathrm{~d}, J=8.0 \mathrm{~Hz}$, $2 \mathrm{H}), 7.14(\mathrm{t}, J=8.6 \mathrm{~Hz}, 2 \mathrm{H}), 2.43(\mathrm{~s}, 3 \mathrm{H}) ;{ }^{13} \mathrm{C}$ NMR $(150$ $\left.\mathrm{MHz}, \mathrm{CDCl}_{3}\right) \delta: 195.0,165.3(\mathrm{~d}, J=252.2 \mathrm{~Hz}, 1 \mathrm{C}), 143.3$, $134.8,134.2,132.5$ (d, $J=9.0 \mathrm{~Hz}, 1 \mathrm{C}), 130.2,129.0,115.4$ (d, $J=21.8 \mathrm{~Hz}, 1 \mathrm{C}), 21.7$.

4-氟-4'- 甲氧基二苯甲酮 (2l) ${ }^{[18]}$ : 白色固体, 产率 88\%. m.p. $98 \sim 99{ }^{\circ} \mathrm{C} ;{ }^{1} \mathrm{H}$ NMR (600 MHz, $\left.\mathrm{CDCl}_{3}\right) \delta$ :
7.79 (dt, $J=8.9,2.6 \mathrm{~Hz}, 4 \mathrm{H}), 7.14$ (t, $J=8.6 \mathrm{~Hz}, 2 \mathrm{H}), 6.96$ (d, $J=8.8 \mathrm{~Hz}, 2 \mathrm{H}), 3.88(\mathrm{~s}, 3 \mathrm{H}) ;{ }^{13} \mathrm{C}$ NMR $(150 \mathrm{MHz}$, $\left.\mathrm{CDCl}_{3}\right) \delta: 194.1,165.1(\mathrm{~d}, J=251.6 \mathrm{~Hz}, 1 \mathrm{C}), 163.3,134.5$, 132.4, 132.3 (d, $J=9.0 \mathrm{~Hz}, 1 \mathrm{C}$ ), 130.0, 115.3 (d, $J=21.8$ $\mathrm{Hz}, 1 \mathrm{C}), 113.7,55.5$.

4-氯苯乙酩 $(2 \mathrm{~m})^{[19]}$ : 白色液体, 产率 $81 \% .{ }^{1} \mathrm{H}$ NMR $\left(600 \mathrm{MHz}, \mathrm{CDCl}_{3}\right) \delta: 7.89$ (d, $\left.J=8.6 \mathrm{~Hz}, 2 \mathrm{H}\right), 7.43$ (d, $J=$ $8.6 \mathrm{~Hz}, 2 \mathrm{H}), 2.59(\mathrm{~s}, 3 \mathrm{H}) ;{ }^{13} \mathrm{C} \mathrm{NMR}\left(150 \mathrm{MHz}, \mathrm{CDCl}_{3}\right) \delta$ : 196.8, 139.6, 135.4, 129.7, 128.9, 26.6.

3-氯苯乙酮 $(2 n)^{[19]}$ : 淡黄色液体, 产率 $86 \% .{ }^{1} \mathrm{H}$ NMR $\left(600 \mathrm{MHz}, \mathrm{CDCl}_{3}\right) \delta: 7.92(\mathrm{t}, J=1.8 \mathrm{~Hz}, 1 \mathrm{H}), 7.83$ (d, $J=7.8 \mathrm{~Hz}, 1 \mathrm{H}), 7.54 \sim 7.52(\mathrm{~m}, 1 \mathrm{H}), 7.41(\mathrm{t}, J=7.9$ $\mathrm{Hz}, 1 \mathrm{H}), 2.60$ (s, 3H); ${ }^{13} \mathrm{C}$ NMR (150 MHz, $\left.\mathrm{CDCl}_{3}\right) \delta$ : 196.7, 138.6, 134.9, 133.0, 130.0, 128.4, 126.4, 26.6.

4-甲基苯乙酮(2o ${ }^{[19]}$ : 无色透明液体，产率 $79 \% .{ }^{1} \mathrm{H}$ NMR $\left(600 \mathrm{MHz}, \mathrm{CDCl}_{3}\right) \delta: 7.85(\mathrm{~d}, J=8.2 \mathrm{~Hz}, 2 \mathrm{H})$, $7.25 \sim 7.24(\mathrm{~m}, 2 \mathrm{H}), 2.56(\mathrm{~s}, 3 \mathrm{H}), 2.40(\mathrm{~s}, 3 \mathrm{H}) ;{ }^{13} \mathrm{C} \mathrm{NMR}$ $\left(150 \mathrm{MHz}, \mathrm{CDCl}_{3}\right) \delta: 197.8,143.9,134.7,129.2,128.4$, 26.5, 21.6.

4-甲氧基苯乙酩 (2p $)^{[19]}$ : 白色固体, 产率 83\%. m.p. $36 \sim 37{ }^{\circ} \mathrm{C} ;{ }^{1} \mathrm{H}$ NMR $\left(600 \mathrm{MHz}, \mathrm{CDCl}_{3}\right) \delta: 7.95 \sim 7.92$ (m, 2H), 6.94 6.92 (m, 2H), $3.86(\mathrm{~s}, 3 \mathrm{H}), 2.55(\mathrm{~s}, 3 \mathrm{H})$; ${ }^{13} \mathrm{C}$ NMR (150 MHz, $\left.\mathrm{CDCl}_{3}\right) \delta: 196.8,163.5,130.6,130.3$, 113.7, 55.5, 26.3.

苯乙酮 $(2 q){ }^{[19]}$ : 浅黄色液体, 产率 $85 \% .{ }^{1} \mathrm{H}$ NMR $\left(600 \mathrm{MHz}, \mathrm{CDCl}_{3}\right) \delta: 7.96 \sim 7.94(\mathrm{~m}, 2 \mathrm{H}), 7.57 \sim 7.54(\mathrm{~m}$, $1 \mathrm{H}), 7.43$ (t, $J=7.8 \mathrm{~Hz}, 2 \mathrm{H}), 2.59$ (s, 3H); ${ }^{13} \mathrm{C}$ NMR $(150$ $\left.\mathrm{MHz}, \mathrm{CDCl}_{3}\right) \delta: 198.2,137.1,133.1,128.6,128.3,26.6$.

2-荎乙酮 $(2 r)^{[20]}$ : 白色固体, 产率 $89 \%$. m.p. 54 $55{ }^{\circ} \mathrm{C} ;{ }^{1} \mathrm{H}$ NMR $\left(600 \mathrm{MHz}, \mathrm{CDCl}_{3}\right) \delta: 8.45(\mathrm{~s}, 1 \mathrm{H}), 8.06 \sim$ 7.99 (m, 1H), 7.95 (d, $J=7.8 \mathrm{~Hz}, 1 \mathrm{H}), 7.87$ (t, $J=8.1 \mathrm{~Hz}$, $2 \mathrm{H}), 7.63 \sim 7.51(\mathrm{~m}, 2 \mathrm{H}), 2.71(\mathrm{~s}, 3 \mathrm{H}) ;{ }^{13} \mathrm{C}$ NMR $(150$ $\left.\mathrm{MHz}, \mathrm{CDCl}_{3}\right) \delta: 198.1,135.6,134.5,132.5,130.2,129.6$, $128.5,128.4,127.8,126.8,123.9,26.7$.

2-甲基二苯甲酮 $(2 \mathbf{v})^{[16]}$ : 无色油状液体, 产率 $63 \%$. ${ }^{1} \mathrm{H}$ NMR (600 MHz, $\left.\mathrm{CDCl}_{3}\right) \delta: 7.85(\mathrm{~d}, J=9.5 \mathrm{~Hz}, 2 \mathrm{H})$, $7.61(\mathrm{t}, J=7.4 \mathrm{~Hz}, 1 \mathrm{H}), 7.48(\mathrm{t}, J=7.8 \mathrm{~Hz}, 2 \mathrm{H}), 7.42(\mathrm{t}$, $J=7.5 \mathrm{~Hz}, 1 \mathrm{H}), 7.36 \sim 7.31(\mathrm{~m}, 2 \mathrm{H}), 7.28(\mathrm{t}, J=7.7 \mathrm{~Hz}$, $1 \mathrm{H}), 2.38(\mathrm{~s}, 3 \mathrm{H}) ;{ }^{13} \mathrm{C} \mathrm{NMR}\left(150 \mathrm{MHz}, \mathrm{CDCl}_{3}\right) \delta: 198.7$, 138.6, 137.8, 136.8, 133.2, 131.1, 130.3, 130.2, 128.6, $128.5,125.3,20.0$.

2,2'-二甲基二苯甲酮 $(\mathbf{2 w})^{[16]}$ : 浅黄色液体, 产率 $31 \%$. ${ }^{1} \mathrm{H}$ NMR (600 MHz, $\left.\mathrm{CDCl}_{3}\right) \delta: 7.42(\mathrm{t}, J=7.5 \mathrm{~Hz}$, 2H), $7.33(\mathrm{dd}, J=17.4,7.7 \mathrm{~Hz}, 4 \mathrm{H}), 7.23(\mathrm{t}, J=7.6 \mathrm{~Hz}$, 2H), $2.48(\mathrm{~s}, 6 \mathrm{H}) ;{ }^{13} \mathrm{C} \mathrm{NMR}\left(150 \mathrm{MHz}, \mathrm{CDCl}_{3}\right) \delta: 200.8$, 
139.0, 138.2, 131.5, 131.1, 130.3, 125.4, 20.7.

辅助材料(Supporting Information) 氧化产物 $\mathbf{2 a} \sim 2 \mathrm{w}$

的氢谱和碳谱. 这些材料可以免费从本刊网站(http:// sioc- journal.cn/)上下载.

\section{References}

[1] (a) Gultekin, M. S.; Çelik, M. Org. Chem. 2004, 8, 1159.

(b) Schröder, M. Chem. Rev. 1980, 80, 187.

(c) Kolb, H. C.; VanNieuwenzhe, M. S.; Sharpless, K. B. Chem. Rev. 1994, 94, 2483.

[2] (a) Zhang, W.; Loebach, J. L.; Wilson, S. R.; Jacobsen, E. N. J. Am. Chem. Soc. 1990, 112, 2801

(b) Irie, R.; Noda, K.; Ito, Y.; Matsumoto, N.; Katsuki, T. Tetrahedron Lett. 1990, 31, 7345 .

(c)Murase, N.; Hoshino, Y.; Oishi, M.; Yamamoto, H. J. Org. Chem. 1999, 64, 338.

(d) Hoshino, Y.; Yamamoto, H. J. Am. Chem. Soc. 2000, 122, 10452.

(e) Makita, N.; Hoshino, Y.; Yamamoto, H. Angew. Chem., Int. Ed. 2003, 42, 941.

(f) Zhang, W.; Yamamoto, H. J. Am. Chem. Soc. 2007, 129, 286.

[3] (a) Criegee, R. Angew. Chem., Int. Ed. Engl. 1975, 14, 745.

(b) Harries, C. Justus Liebigs Ann. Chem. 1905, 343, 311.

[4] (a) Koike, K.; Inoue, G.; Fukuda, T. J. Chem. Eng. Jpn. 1999, 32, 295.

(b) Dorofeev, S. B.; Eletskii, A. V.; Smimov, B. M. Sov. Phys. Dokl. 1981, 26, 318 .

(c) Ogle, R. A.; Schumacher, J. L. Process Saf. Prog. 1998, 17, 127.

[5] Kaneda, K.; Haruna, S.; Imanaka, T.; Kawamoto, K. J. Chem. Soc., Chem. Commun. 1990, 1467.

[6] (a) Travis, B. R.; Narayan, R. S.; Borhan, B. J. Am. Chem. Soc. 2002, 124, 3824. (b) Yu, W.; Mei, Y.; Kang, Y.; Hua, Z.; Jin, Z. Org. Lett. 2004, 6 , 3217.

(c) Whitehead, D. C.; Travis, B. R.; Borhan, B. Tetrahedron Lett. 2006, 47, 3797.

(d) Nicolaou, K. C.; Adsool, V. A.; Hale, C. R. H. Org. Lett. 2010, $12,1552$.

[7] Arasasingham, R. D.; He, G.-X.; Bruice, T. C. J. Am. Chem. Soc. 1993, 115, 7985

[8] Sato, K.; Aoki, L.; Noyori, R. Science 1998, 281, 1646.

[9] Hermann, W. A.; Weskamp, T.; Zoller, J. P.; Fischer, R. W. J. Mol. Catal. A: Chem. 2000, 153, 49.

[10] Wang, A.; Jiang, H. J. Org. Chem. 2010, 75, 2321.

[11] (a) Shaikh, T. M.; Hong, F.-E. Adv. Synth. Catal. 2011, 353, 1491. (b) Ma, S.; Liu, J.; Li, S.; Chen, B.; Cheng, J.; Kuang, J.; Liu, Y.; Wan, B.; Wang, Y.; Ye, J.; Yu, Q.; Yuan, W.; Yu, S. Adv. Synth. Catal. 2011, 353, 1005.

[12] Xing, D.; Guan, B.; Cai, G.; Fang, Z.; Yang, L.; Shi, Z. Org. Lett. 2006, 8,693

[13] (a) Hossain, M. M.; Shyu, S.-G. Tetrahedron 2014, 70, 251.

(b) Gu, L.; Liu, J.; Zhang, H. Chin. J. Chem. 2014, 32, 1267.

[14] (a) Miyamoto, K.; Tada, N.; Ochiai, M. J. Am. Chem. Soc. 2007, 129, 2772.

(b) Miyamoto, K.; Sei, Y.; Yamaguchi, K.; Ochiai, M. J. Am. Chem. Soc. 2009, 131, 1382.

[15] Hossain, M. M.; Huang, W.-K.; Chen, H.-J.; Wang, P.-H.; Shyu, S.-G. Green Chem. 2014, 16, 3013.

[16] Schmink, Jason R.; Krska, Shane W. J. Am. Chem. Soc. 2011, 133, 19574.

[17] Miao, T.; Wang, G.-W. Chem. Commun. 2011, 47, 9501.

[18] Liao, Y.-X.; Hu, Q.-S. J. Org. Chem. 2010, 75, 6986.

[19] Ruan, J.-W.; Li, X.-M.; Saidi, O.; Xiao, J.-L. J. Am. Chem. Soc. 2008, 130, 2424

[20] Dohi, T.; Fukushima, K.-I.; Kamitanaka, T.; Morimoto, K.; Takenaga, N.; Kita, Y. Green Chem. 2014, 1493.

[21] Joshi, K. C.; Giri, S. J. Ind. Chem. Soc. 1963, 40, 42.

(Li, L.; Fan, Y.) 